\title{
Announcement and Rules
}

\section{C.E. Alken Foundation Council}

Bern, January 2001

The C.E. Alken Foundation supports clinical and experimental research through an annual prize for outstanding scientific work.

The C.E. Alken Prize is awarded for the best unpublished scientific work in the field of urology and may be divided. The prize comprises a certificate and the sum of SFr. 10,000.

Manuscripts can be subtmitted in either English or German. Seven copies of the manuscript should be sent to the following address and marked for the attention of Mr. E. Hauser, Advocate:

C.E. Alken Foundation

Dr. F. Kellerhals \& Partner

Marktgasse 55

CH-3011 Bern (Switzerland)

The deadline for submission of manuscripts is September 1, 2001. Each paper has to be marked with a code word and must not include the name of the author. An additional sealed envelope (marked on the outside with the code word) has to be enclosed and must contain the following:

- Curriculum vitae of the author (max. 3 pages).

- Structured summary of the manuscript (aim of study, material/ methods, results, conclusions).

The award winner will be determined by the Foundation Council, whose decisions is final. 